\title{
Ferdinand de Saussure et Eugenio Coseriu : propositions pour une linguistique intégraliste
}

\author{
Clemilton Lopes Pinheiro ${ }^{1 *}$ \\ ${ }^{1}$ Université fédérale de Rio Grande do Norte (UFRN), 59078-970, Natal, Brésil
}

\begin{abstract}
Résumé. Dans ce article, nous nous proposons de réaliser une discussion épistémologique sur le travail de Ferdinand de Saussure et celui d'Eugenio Coseriu ayant comme objectif celui de mettre en relation les positionnements face à la notion de linguistique intégrale. Pour atteindre cet objectif, nous avons procédé à une analyse interprétative de quelques œuvres. Du côté de Coseriu nous avons cherché des œuvres dans lesquelles le thème est explicitement traité. Du côté de Saussure, nous avons eu recours aux manuscrit de Constantin du troisième cours, quelques travaux interprétatifs de ce manuscrit, aussi bien que quelques travaux interprétatifs des manuscrits sur les anagrammes et les légendes. Nous défendons que les réflexions de Saussure et celles de Coseriu indiquent la nécessité d'une linguistique intégrale, c'est-à-dire, une linguistique qui doit rendre compte des différents aspects impliqués dans le fonctionnement complexe du langage, en ordonnant ces aspects dans une marque homogène et unitaire. Ce raisonnement nous a par conséquent amené à penser, que pour bien comprendre le langage, il est nécessaire de préciser des aspects différents et des disciplines différentes.
\end{abstract}

\begin{abstract}
Ferdinand de Saussure and Eugenio Coseriu : proposals for an integralist linguist. In this paper, we propose to hold an epistemological discussion of the work of Ferdinand de Saussure and Eugenio Coseriu with the aim of relating the positions regarding the notion of integral linguistics. In order to achieve this goal, we conducted an interpretative analysis of some works. On the side of Coseriu, we look for works in which the theme is explicitly dealt with. On Saussure's side, we look at Constantin's notebooks of the third course, some interpretative works of this manuscript, and some interpretative works of the manuscripts on anagrams and legends. We argue that Saussure's and Coseriu's reflections point to the need for an integral linguistics, that is, a linguistics that must account for the different aspects involved in the complex functioning of language, ordering these aspects in a homogeneous and unitary framework. This reasoning leads us to think, therefore, that, in order to understand language well, it is necessary to specify different aspects and different disciplines.
\end{abstract}

\section{Introduction}

Ferdinand de Saussure est considéré comme étant le fondateur de la linguistique moderne. L'importance de ses réflexions sur le langage reste reconnue, un siècle après la publication du Cours de Linguistique Générale (CLG), en 1916, par des linguistes des plus différentes orientations théoriques, qui ont également interprété avec rigueur sa pensée, en

*clemiltonpinheiro@hotmail.com 
altérant ou suggérant des développements. Eugenio Coseriu, incontestablement, un autre éminent linguiste de la scène contemporaine, a été un lecteur assidu et perspicace de Saussure. Les chercheurs ont l'habitude de dire qu'une bonne partie de l'œuvre fondamentale de Coseriu, principalement celle des années 50, provient d'une confrontation avec les idées de Saussure. Dans le célèbre essai Sistema, norma y habla, par exemple, Coseriu a développé la notion théorique de norme, et s'est écarté de Saussure, en établissant la distinction entre langue, système fonctionnel, et norme, système normal. En effet, Coseriu a mis en évidence, tout au long de son œuvre considérable, que la linguistique doit dépasser le structuralisme, et donc, aller au-delà de Saussure. Dans ce sens, il a proposé une « linguistique intégrale ».

Cette opposition entre Saussure et Coseriu, semble, cependant se défaire, en étant considérée comme l'examen du corpus saussurien, qui suivant Bronckart, Bulea et Bota (2010:12), «conduit d'abord à récuser définitivement un ensemble de clichés, soit directement, issu du CLG, soit élaborés puis vulgarisés sur la base d'une lecture trop rapide de cet ouvrage ». Bronckart, Bulea et Bota (2010 : 13) nient, ainsi, l'existence de deux Saussure. Il n'y en a qu'un, celui qui a essayé de construire une linguistique suivant une perspective qui combine «une totale exigence théorique-épistémologique avec une véritable prise en considération (techniquement très armée) de ces realia que constituent les textes effectifs et les langues naturelles ». Dans ce sens, nous pouvons voir également dans Saussure une perspective intégraliste de linguistique.

Ce travail cherche à explorer cette discussion. Nous nous proposons de réaliser une réflexion épistémologique sur le travail des deux linguistes ayant pour objectif de mettre en relation les positionnements sur la notion de linguistique générale. Pour atteindre cet objectif, nous avons procédé à une analyse interprétative de quelques œuvres. Du côté de Coseriu, nous avons cherché des oeuvres dans lesquelles le thème est explicitement abordé. Du côté de Saussure, nous avons eu recours aux cahiers du troisième cours (Constantin, 2005), quelques travaux interprétatifs de ce manuscrit, et quelques travaux interprétatifs des manuscrits sur les anagrammes et les légendes.

\section{Le projet intégraliste de Saussure}

Le Suisse Ferdinand de Saussure, né à Genève, en 1857, est, indiscutablement, le nom le plus fréquemment évoqué quand on aborde la linguistique du $\mathrm{XX}^{\circ}$ siècle. Ses premiers travaux, déjà publiés au $\mathrm{XIX}^{\circ}$ siècle, ont abordé la grammaire comparative des langues indo-européennes, conforme au paradigme linguistique hégémonique de l'époque. Au moyen d'une exploration des notes de cours prises par quelques élèves durant les trois derniers cours que Saussure a dispensés sur la linguistique générale, à l'université de Genève, de 1907 à 1911, Charles Bally et Albert Sechehaye ont organisé le CLG (Saussure, 1916), qui est devenu un texte base dans ce domaine.

Suivant de près le CLG, la divulgation des idées de Saussure a focalisé la présentation de dichotomies (langue/parole, syntagme/paradigme, forme/substance, signifié/signifiant, synchronie/diachronie) autour desquelles a été délimité l'objet d'étude primordial de la linguistique: la langue. C'est ainsi que le CLG représente un moment décisif dans l'histoire de la linguistique en tant que Science, comme le signalent (Colombat, Fournier, Puech, 2010:25): «Il demeure enfin, aujourd'hui même, un texte d'initiation à la linguistique dans de nombreux cursus universitaires dans le monde entier ».

Quelques réserves sur le travail de Bally et de Sechehaye, comme une reconstruction basée sur des sources hétérogènes et fragmentées de la pensée de Saussure, ont été 
présentées par quelques-uns de ses anciens élèves, mais sont restées sans écho pendant beaucoup de temps (Bouquet, 2010). Les travaux critiques de Godel, Engler et De Mauro, à partir de sources manuscrites, principalement les cahiers des élèves, ont également commencé à montrer quelques problèmes dus à le réinterprétation et à l'appropriation de la pensée saussurienne qu'il a présentée lui-même.

En 1996, pendant des travaux dans l'ancienne résidence de la famille Saussure à Genève, un ensemble de feuilles manuscrites par le propre Saussure a été découvert. Ce matériel, cédé à la bibliothèque de Genève, a intégré d'autres œuvres déjà existantes et a été publié sous le titre : Écrits de linguistique générale (Saussure, 2002). À partir de là, on a commencé à développer une révision fondamentale sur de nombreux points abordés dans le CLG et donc de l'image que jusqu'alors on se faisait de Saussure et de sa propre conception du langage. Selon Jäger (2013), dans ses manuscrits, Saussure indique les «points délicats » de la théorie de la langue et du signe dont la discussion devrait apporter des conséquences significatives pour une refondation de la linguistique.

Ces textes appartiennent par conséquent sans aucun doute au contexte d'une réflexion épistémologique sur le problème de la langue, qui représente pour Saussure la condition indispensable pour une refondation possible de la linguistique - refondation à laquelle il contribua par des réflexions fondamentales dans les notes de l'orangerie, qui portent le titre « la science du langage ». Avec ce titre, il avait à l'évidence son utopie en vue, l'idée de cette linguistique future dont l'orientation devait être préparée par ses réflexions fragmentaires et aphoristiques (Jäger, $2013: 55$ )

Dans ce sens, beaucoup de chercheurs ont parlé d'une théorie saussurienne du langage comme étant un projet plus ample qui surpasse l'ensemble des concepts présents dans le CLG. C'est là une idée défendue, par exemple, par Bronckart, Bulea, Bota (2010:11) :

[...] il apparaît que Saussure n'a poursuivi, depuis le Mémoire et jusqu'aux derniers cours, qu'un seul et même projet d'élaboration d'une science linguistique, qui saisirait les formes d'organisation des langues dans la totalité de leurs aspects, et qui prendrait notament à bras le corps la problématique de la transformation des faits langagiers tout autant que celle du statut intégralemente psychique des signes.

Nous n'entrerons pas, ici, dans l'essentiel des différentes visions autour des manuscrits de Saussure ni dans les différents traitements que ces données ont reçu des chercheurs. Notre intention n'est que de proposer que le projet saussurien pour une science du langage possède la nature integraliste, c'est-à-dire, qu'il s'agit d'un projet basé dans la perspective suivant laquelle le langage présente des composants et des propriétés distincts et l'étude intégrale du langage doit prendre en compte, à partir d'un abordage cohérent avec sa nature, chaque composant et chaque propriété séparément. Le langage étant un phénomène complexe, multi-forme, hétéroclite, «Il est à cheval sur des domaines divers (domaine physique, psychique, ou encore : domaine individuel, social) » (Constantin, 2005 :214).

La préparation que Saussure présente pour le Cours III (Constantin, 2005) nous permet d'observer trois domaines ou aspects différents qui doivent être considérés dans le traitement du langage : les langues, la langue et l'exercice individuel du langage. Par 
conséquent, la langue en tant que système de signes est à peine un des composants, et comme le signale Gambarara (2005:30), elle est en deuxième position dans la préparation du cours.

Pensons seulement que le plan du IIIe cours prévoit d'emblée une première partie Les langues, et seulement dans un deuxième temps la section consacrée à La langue. Or, le CLG donne la première place dans l'exposition à ce qui était la deuxième partie de Saussure, et rejette à la fin du livre, dans un chapitre qui n'attire pas l'attention, les questions que Saussure considérait comme préliminaires.

Le premier plan du projet intégraliste de Saussure, par conséquent, est celui des langues, c'est-à dire, le plan des propriétés ou des régularités communes à toutes les langues:

Il faut ajouter que le langage de l'ensemble de l'humanité se manifeste par une diversité de langues infinie: la langue est le produit d'une société, mais les différentes sociétés n'ont pas la même langue. D'où vient cette diversité? Tantôt c'est une diversité relative, tantôt c'est une diversité absolue, mais enfin nous avons trouvé l'objet concret dans ce produit que l'on peut supposer déposé dans le cerveau de chacun. Mais ce produit, suivant qu'on se place à tel endroit du globe, est différent; la $<$ chose $>$ donnée, ce n'est pas seulement la langue mais les langues. Et le linguiste est dans l'impossibilité d'étudier autre chose au début que la diversité des langues. Il doit étudier d'abord les langues, le plus possible de langues; il doit étendre son horizon autant qu'il le peut. C'est ainsi que nous procéderons. Par l'étude, l'observation de ces langues, il pourra tirer des traits généraux, il retiendra tout ce qui lui paraît essentiel et universel, pour laisser de côté le particulier et l'accidentel. (Constantin, $2005: 89$ ).

Comme ce que l'on aperçoit dans les langues est un phénomène continu dans le temps et dans l'espace, il ne peut pas y avoir de division nette entre, par exemple, une langue «actuelle» et une langue "ancienne» ou une langue «mère » et une langue «fille». Ainsi, Saussure a dédié à ce domaine, celui des langues, la première partie du Cours III, 15 leçons au total, pendant plus ou moins deux mois, selon Gambarara (2005). Dans ces leçons, le thème abordé a été la diversité géographique des langues en tant que résultat et témoignage de leur évolution, et l'écriture, considéré également comme témoignage indispensable des états passés des langues. Se basant sur les analyses des différences et des similitudes entre les langues les plus différentes dans le temps et dans l'espace, ce que Saussure a cherché à montrer, en fait, ce sont les causes par lesquelles les langues évoluent surtout en fonction du temps. Comment pouvons-nous savoir si une langue ne sera pas identique après un certain temps? «D'où le savons-nous ? - Parce que c'est l'expérience universelle » (Constantin, $2005: 112)$.

Il nous parait encore, dans ce cas, qu'au-delà de l'explication des causes de la diversité des langues, il existe une recherche pour la compréhension d'un phénomène qui présente un caractère proprement ontologique, dont la réalité est unique et universelle. Le focus de la linguistique, dans ce domaine, parait être, par conséquent, les mécanismes communs, l'unicité ontologique, universellement présente dans toutes les langues. 
Il reste un intéressant terrain de comparaison entre langues n'ayant aucune parenté visible, aucune origine commune: c'est le travail de comparaison de l'organisme grammatical, <comparaison des différents contrats possibles entre pensée et langue $>$. Il est possible que des langues nullement parentes réalisent un mécanisme grammatical tout à fait semblable (Constantin, 2005 : 99)

Le deuxième plan est la langue conçue comme un système de signes organisé par une communauté déterminée de sujets parlants, un système où toutes les unités sont solidaires et la valeur de l'une d'elles est donnée par la présence et l'interaction avec d'autres.

L'intérêt de l'attention du linguiste, dans ce plan, est, par conséquent, ce système qui a constitué une entité autonome de dépendances internes. Ainsi, ce système ne peut être étudié qu'en synchronie, car les relations d'unités coexistantes qui le forment sont perçues par une unique conscience collective. De ce principe, découle la proposition méthodologique structuraliste de description linguistique, également connue sous le nom de linguistique de la langue, dans laquelle s'est concentrée l'attention des linguistes pendant très longtemps.

Finalement, le troisième plan, celui de l'exercice individuel du langage, peut être déduit des réflexions que Saussure a fait sur la notion de discours. Beaucoup d'analystes ont défendu que l'opposition langue/parole englobe une discussion beaucoup plus vaste que la simple opposition présente dans le CLG entre niveau social et niveau individuel du langage. Saussure a déclaré à plusieurs reprises que la langue est, de fait, sociale, mais la parole est en même temps sociale et individuelle. La langue est un ensemble de potentialités dont la réalisation se produit par le biais d'un acte, la parole, qui peut être singulier, mais également social, car il est soumis à des cohésions sociales. Saussure se positionne aussi de façon différente face au caractère secondaire de la parole. Pour lui, la parole est la source de création de nouvelles formes de la langue, et, ainsi, inverse le statut de la parole, qui va être présentée en tant qu'entité active en opposition à la langue, entité passive (Bota et Bronckart, 2010).

Bien que ces révisions sur le statut de la parole indiquent le fait que le discours n'a jamais été étranger à la réflexion linguistique de Saussure, d'autres travaux indiquent que Saussure a vraiment pensé à un concept de discours différent de celui de la parole, comme le souligne Testenoire (2016:125):

Ce qu'il importe surtout de noter c'est que les efforts de certains critiques, pas toujours philologiquement bien informés, pour révéler, à partir de la note " sur le discours », un concept saussurien de discours totalement disjoint de celui de parole repose avant tout sur une approche immanente des textes.

De la même façon, les objectifs apparents de l'étude des anagrammes et des légendes ont semblé révéler un projet que visait à réfléchir sur une autre dimension du langage, comme la dimension des textes et des discours. Selon Pinheiro (2006), dans les manuscrits sur les légendes, Saussure rejette la proposition d'analyser les légendes sous la perspective de la vérité ou de la fausseté des faits, en prenant comme base l'histoire. Au contraire, la légende est prise comme un texte ou un discours particulier dont le sens doit être compris avec une base dans différents facteurs. Au-delà de cela, toujours selon Pinheiro (2006), en cherchant à comprendre les conditions d'urgence et de stabilisation des légendes, Saussure souligne l'importance des traits singuliers du langage. 
Nous avons vu par ailleurs que les questions soulevées par Saussure dans les manuscrits sur les légendes suggèrent qu'il considère le texte dans son individualité, comme un événement singulier dont il tente d'expliquer le sens. Dans cette perspective, le texte n'est pas considéré comme un fait de langue en tant que système historiquement déterminé. [...] Chez Saussure, le signe linguistique est un type de signe parmi d'autres mais dont la spécificité et la complexité du fonctionnement fait de la langue un système sémiologique à part. [...] La légende et la langue sont considérées comme deux objets de la sémiologie, chacune portant un type de contenu spécifique qui nécessite, bien évidemment, des cadres théoriques distincts. (Pinheiro, $2016: 191-92$ )

Pour Pinheiro (2016), par conséquent, il semble que Saussure a pensé pouvoir traiter également les propriétés des textes/discours comme une dimension affectée à l'exercice individuel du langage.

Quoique cela ne puisse être qu'une supposition, en se basant dans les sources disponibles ainsi que dans les études déjà réalisées, nous pouvons penser que, dans le projet de Saussure, il y avait une idée intégraliste de linguistique suivant laquelle l'étude du langage s'organise en trois plans qui doivent être articulés, chacun d'eux relationné à une dimension: celle des langues (les propriétés communes à toutes les langues), celle de la langue (les propriétés d'une langue particulière, en tant que système de valeurs accessible à une même communauté dans un espace et un moment déterminés), et de l'expression individuelle (propriétés singulières de l'utilisation du langage révélées dans les textes/discours). Nous devons admettre encore que le deuxième plan a été le plus solidement abordé, et même en tant que principal objet du CLG. Les deux autres plans n'ont pas été systématisés, de même que n'a pas été démontrée l'articulation qui convient entre les trois plans.

Selon Jäger (2013 : 69), Saussure avait une conception sémiologique de langue, mais n'est pas arrivé à la développer de forme systématique dans un texte:

Saussure développa bien plutôt ses réflexions par l'intermédiaire d'un ductus aphoristique et fragmentaire, celui de ce que l'on pourrait nommer un journal de pensée, qu'il réalisa avant tout dans les trois groupes de notes, les «Notes inédites », les notes sur l'accent lituanien et les notes de l'orangerie, qui documentent pour ainsi dire un laboratoire d'un autre genre : l'espace de pensée dans lequel Saussure a développé pour lui-même sa conception sémiologique de la langue.

Comme cette conception était encore dans cet « espace de pensée », il est possible de supposer que ce soit la raison pour laquelle Saussure refusait de publier ses idées et, d'ailleurs, resistait à donner des cours de linguistique générale, comme le défendent Bronckart, Bulea, Bota (2010 : 9): "Cette ambition d'une linguistique véritablement générale s'est cependant, et bien évidemment, heurtée à de multiples obstacles, d'ordre épistémologique, conceptuel et méthodologique ». Comme il n'y a eu qu'un seul des plans qui avait été conçu, la proposition d'une linguistique intégraliste courait le risque d'être incomprise, si elle était présentée sans la conception complète des autres plans. La réflexion de Saussure sur ses trois objets n'a pas été continue, ni stable, elle allait et venait. Il paraîtrait, si nous considerons l'opinion de Quijano (2005), qu'il n'a pas eu le temps de vie 
nécessaire pour systématiser le cadre théorique d'une linguistique intégraliste, à laquelle il aspirait.

Aussi pourrait-on voir dans ce parcours une simple répétition de la démarche du chercheur. Il n'en est rien. Durant trente ans, le doute a été le moteur de l'exploration, mais il tournait un peu à vide: Saussure laissait tomber une recherche sur un point de butée, l'intonation lituanienne, par exemple, et commençait d'autres recherches sur des terrains différents, les légendes germaniques, par exemple, abandonnées à leur tour pour faire place aux anagrammes, sans que le chercheur ait pu faire le lien entre ces divers sujets. Un peu comme ces personnes qui vivent des histoires d'amour successives avec des partenaires différents mais qui butent toujours sur le même point de conflit.(Quijano, 2005 : 47)

\section{Le projet intégraliste de Coseriu}

Eugenio Coseriu n'a pas été aussi célèbre que Saussure, mais il n'a pourtant pas été moins important pour la pensée linguistique moderne. Il a fait ses études en Roumanie puis en Italie, et est devenu professeur de linguistique générale et indo-européenne, à l'université de Montevideo. En 1963, il a été nommé professeur de linguistique roumaine et générale, à Tübingen en Allemagne. Pendant les années 70, l'école coseriane de Tübingen est devenue l'une des plus prestigieuses en linguistique roumaine, exerçant une grande influence, principalement dans le domaine de la linguistique générale et de la philologie du langage.

Coseriu a développé les principes fondamentaux de sa théorie du langage en se basant encore dans la linguistique structurelle, mais il a cependant dominé presque toutes les thématiques de la linguistique générale et une quantité notable d'études philologiques de langues particulières. L'extension thématique de son œuvre est une manifestation externe de sa conception personnelle autour de ce que sont le langage et la propre linguistique. La proposition de Coseriu était de pouvoir comprendre toute la réalité de la langue et de l'intégrer systématiquement dans un modèle épistémologique fonctionnel. Selon Kabatek (2004), Coseriu est fréquemment vu comme un pur structuraliste, mais il défend que ses travaux ont toujours suivi deux types d'objectifs.

D'un côté, celui de prendre au sérieux la linguistique structuraliste dans toute son extension, c'est-à-dire, recouvrir tous les domaines de la langue; d'un autre côté, celui de montrer ses limites, car, pour Coseriu, le structuralisme n'offre qu'une vision partielle du langage, en laissant entre parenthèses une série de faits (exclus par les célèbres "sept distinctions") pour atteindre son objectif. Le devoir de la "linguistique intégrale" est, alors, d'aller également audelà du structuralisme et réintégrer tout ce qu'il a exclus. (Kabatek, 2004:487).

De fait, Coseriu (1982) défend la nécessité d'aller au-delà du structuralisme. Pour lui, « aller au-delà » ne signifie pas abolir, mais reconnaître tout ce qu'il y a de valide comme point de vue, comme concept et résultats positifs en ce qui concerne la connaissance du langage et des langues, et réintégrer tout ce qu'il a exclus : «Il s'agit de rendre compte de 
toute la compétence du sujet linguistique, de toutes les connaissances que le sujet linguistique met en œuvre au moment de parler» (Coseriu, 1982 : 168). Dans ce sens, Coseriu fait remarquer la perspective d'une linguistique intégrale, c'est-à-dire, une linguistique qui admet différents points de vue, autour du même problème. L'idée d'une linguistique intégrale a été citée par Coseriu, dans certains travaux, surtout quand il se positionnait sur le rôle des différents domaines de la linguistique. Selon Hassler (2015:31),

Coseriu s'est exprimé plusieurs fois sur l'état actuel de la linguistique. Il semblait être pessimiste à l'égard de la capacité des linguistes à regarder d'un point de vue critique leur propre travail. Le premier pas vers une linguistique intégrative serait donc celui de montrer le travail effectué et les limites de chaque méthode.

Toujours selon Hassler (2015:29), Coseriu a réalisé ce travail dans le cadre de la sémantique structurelle. Suivant le postulat d'une linguistique intégrale, il «cherche à réintégrer les études qu'il avait éliminées délibérément de la sémantique structurelle: la métalangue, le discours répété et la variation linguistique ».

Coseriu a également parlé de la linguistique intégrale dans des entrevues, comme celle accordée à Johanes Kabatek et Adolfo Murguia, à l'université de Tübingen (Kabatek; Murguia, 1997). Dans l'entrevue, Adolfo Murguia a demandé à Coseriu, par exemple, s'il acceptait le terme linguistique intégrale pour sa théorie. Coseriu l'a confirmé et l'a justifié en se basant sur le présupposé basique qu'il a établi pour la recherche linguistique.

Cette idée de linguistique intégrale s'établit sur le présupposé de ce que le langage présente trois niveaux autonomes : le niveau universel ou niveau du parler en général, le niveau historique des langues, et le niveau individuel des textes. Pour Coseriu (1984:37), chacun de ces niveaux correspond à un des plans de la linguistique intégrale, qu'il définit comme «cette linguistique qui se propose de rendre compte du savoir que le parlant mobilise quand il parle et ordonner les faits vérifiés à cet égard dans une marque homogène et unitaire ».

Le niveau universel vérifie les phénomènes communs à toutes les langues. La première propriété universelle des langues est leur caractère signifié, c'est-à-dire, la possibilité de se référer à quelque chose qui ne s'identifie pas à elle-même. La comparaison entre les langues fournit une évidence pour cette propriété : différentes langues correspondent à des configurations distinctes pour une même réalité extralinguistique. Certaines activités en relation avec le langage, comme la traduction, par exemple, sont seulement possibles à partir du présupposé que différentes langues peuvent se référer à une même réalité et le font d'une façon différenciée. La deuxième propriété que Coseriu a attribuée au niveau universel du langage est la faculté universelle de parler, qui n'est pas déterminée historiquement. "Il s'agit d'un savoir parler qui ne coïncide pas simplement avec le savoir parler allemand, français, etc. mais qui vaut pour toute langue et pour tout langage » (Coseriu, 2007 : 131).

Le deuxième niveau autonome du parler est le niveau historique des langues (le plan des langues particulières). Selon Coseriu (2007 : 132), «personne ne discute que les langues possèdent des structures diverses, des grammaires distinctes, un lexique structuré de façon différente, des contenus de mots configurés également de façon distincte ».

Le troisième niveau, le plan du texte, est le plan individuel du langage, c'est-à-dire, le langage en tant que réalisation d'un individu et le langage réalisé dans une circonstance déterminée. En d'autres mots, il s'agit du plan dans lequel se trouvent les discours, c'est-à- 
dire, les actes linguistiques ou la série d'actes linguistiques de quelqu'un, dans une situation déterminée.

Ces trois plans fonctionnent, ensemble, dans tout acte de langage : le niveau du langage en général, qui vaut pour n'importe quelle langue, le niveau des langues, qui se réfère à l'organisation d'une langue déterminée, et, d'une certaine manière, d'une langue historique, et le niveau du texte. Chacun de ces niveaux a son autonomie, ses propres fonctions, et, le plus important, un type de contenu propre : désignation, signification et sens. Ces types de contenu sont considérés d'une façon isolée, juste méthodologiquement, car, tout discours, présente, en même temps, une désignation, une signification et un sens.

Coseriu, défend, alors, l'existence de trois orientations pour la linguistique, qui correspondent, en essence, à chacun des trois plans, et par conséquent, à chacune des trois séries de fonctions et chacun des trois types de contenu. Il y a ainsi la linguistique qui correspond au plan du langage, aux fonctions du langage et à la désignation. La linguistique qui correspond au plan des langues, au plan historique, aux fonctions propres des langues, et à la signification. Et, finalement, la linguistique qui correspond au plan du discours ou du texte, au sens. En d'autres termes, chacune de ces linguistiques a nécessairement un objet propre, et ne peut que développer, de façon cohérente, des méthodes et des techniques pour la description et l'interprétation de cet objet en particulier. L'auteur défend encore la complémentarité de ces trois projets, car pour que nous comprenions le langage dans toute sa dimension, nous avons effectivement besoin d'une description onomasiologique, d'une description sémasiologique, et également d'une herméneutique du sens.

Comme chaque linguistique correspond à un plan déterminé, elle a ses respectives conséquences, et les problèmes sont spécifiques, l'un ne peut pas résoudre les problèmes de l'autre. Comme les problèmes sont spécifiques, dans la linguistique intégrale, qui a comme intention, celle d'atteindre une connaissance globale du langage, chacune des linguistiques avec ses points de vue respectifs a sa propre place.

Désormais, la grammaire qui concerne nos trois niveaux sera, respectivement: grammaire générale (et non "grammaire universelle") - de toutes les langues - chose impossible et absurde, mais théorie grammaticale : grammaire dont l'intention consiste à définir des catégories verbales - "parties du discours" et les catégories, fonctions et les procédés grammaticaux), grammaire descriptive (de telle ou telle langue en particulier) et analyse grammaticale (du texte donné). (Coseriu, 1980 : 98).

Coseriu (1980) défend que la linguistique, aussi bien la traditionnelle que la moderne, a été linguistique des langues, ou c'est-à-dire, les linguistes ont concentré une grande partie de leur travail pour le niveau historique des langues.Vis-à-vis de la linguistique du langage, également pour Coseriu (1982a : 35), elle n'est pas encore constituée : « quand je dis que la linguistique du langage n'existe pas, je dis qu'elle n'existe pas comme linguistique constituée, parce qu'indirectement, dans les introductions à la linguistique, on fait allusion à cette connaissance des choses que la parole signifie ». De la même façon, il défend que le plan du sens devrait être davantage étudié : «malheureusement, d'une linguistique du sens ne peuvent être vues ébauchées, pour l'instant, que les intentions » (Coseriu, $1980: 100)$.

Dans la vision de Kabatek (2003 : 98), la linguistique intégrale est « une proposition hautement sérieuse et de grande envergure, qui propose une espèce de marque générale pour la recherche linguistique et pour le développement d'une conscience sur le lieu et la 
relation de chaque discipline dans l'ensemble des disciplines ». Pour avoir des arguments en faveur de cette position, Kabatek (2003) se basait sur le fait qu'actuellement, très souvent, on est confronté à une conception trompeuse de spécialisation suivant laquelle la connaissance d'un seul domaine, ou d'un ensemble fait abstraction de la totale ignorance des autres. Au contraire l'auteur défend que «plus le degré de séparation des disciplines est grand, plus la connaissance sur l'endroit qu'elles occupent dans l'ensemble devrait être grand » (Kabatek, 2003 : 98).

Dans la linguistique de nos jours, la proposition d'une linguistique intégrale dans les termes cosériens n'est pas très connue. Dans une entrevue avec Rubo (2017), Kabatek communique que, dans beaucoup de pays, le terme "linguistique intégrale » n'a eu aucun succès, quoique l'idée subjacente soit présente dans différentes écoles. «Je ne crois pas qu'il existe, en Allemagne, quelqu'un qui se définisse comme intégraliste ». En Roumanie, d'un autre côté, mais spécifiquement à l'université Babes-Bolyai, à Cluj Napoca, il existe le Centre d'études intégralistes où sont développés beaucoup de projets suivant cette perspective et où l'on assume l'influence et l'héritage cosérien. Selon Oancea et Obrocea (2013 : 198), la caractéristique plus importante du Centre d'études integralistes de Cluj est l'étude de toutes les étapes de l'intégralisme, «dans une recherche vraiment intégrale ». Cette recherche présuppose cinq paramètres essentiels qui forment un pentagone de l'intégralisme linguistique :

1. a fondation de la linguistique entière sur un fondement épistémologique antipositiviste; 2 . le changement radical de perspective dans l'approche et la création de la linguistique, sur cette base, comme science intégrale de la parole, non seulement des langues; 3. la définition de l'objet de la nouvelle linguistique comme étant la "fonction significtive », à savoir la création de signifiés et/ou la «compétence linguistique», dans le sens consérien; 4. la définition des sous-domaines fondamentaux ( la parole en géneral », les langues, le texte), correspondant aux trois niveaux de compétence (élocutionnelle, idiomatique, expressive), et aux trois couches du contenu (désignation, signification, sens) ; 5 . le développement, dans ce contexte, des recherches sytématiques essentiellement descriptives ou empiriques.

\section{Conclusion}

Les réflexions de Saussure et de Coseriu concernant la constitution de la linguistique semblent indiquer la nécessité d'une linguistique intégrale, c'est-à-dire, une linguistique qui doit rendre compte des différents aspects qui sont en rapport avec le fonctionnement complexe du langage, en ordonnant ces aspects dans une marque homogène et unitaire. Ce raisonnement nous amène à penser, par conséquent, que pour bien comprendre le langage, il est nécessaire de préciser des aspects différents et des disciplines différentes.

Selon Bronckart, Bulea et Bota (2010: 7), en dépit de toute la reconnaissance et la célébrité, l'œuvre de Saussure n'est pas encore bien comprise, et « la dimension proprement révolutionnaire reste largement ignorée ». Nous pouvons étendre un raisonnement identique vis-à-vis de Coseriu. Le grand potentiel de développement de ses travaux n'a pas encore reçu l'attention qu'il mérite.

À notre avis, reprendre les propositions de Saussure et de Coseriu a constitué une entreprise fondamentale pour l'expansion et l'approfondissement des études du langage 
dans l'actualité. Il ne s'agit pas de récupérer l'histoire de la pensée de ces auteurs, mais de découvrir leurs racines, ayant pour objectif les portées et les limites de leurs idées dans le développement des perspectives théoriques et méthodologiques pour la linguistique.

\section{Références bibliographiques}

Bota, C.; Bronckart, J-P. (2010). Dynamique et socialité des faits langagiers. In : Bronckart, J-P, Bulea, E. \& Bota, C. (éds). Le projet de Ferdinand de Saussure. Genève, Droz, 193-213.

Bouquet, S. (2010) Du pseudo-Saussure aux textes saussuriens originaux. In: Bronckar, J-P, Bulea, E. \& Bota, C. (éds). Le projet de Ferdinand de Saussure. Genève, Droz, 31-48.

Bronckart, J-P, Bulea, E. \& Bota, C. (2010). Pour um réexamen du projet saussurien. In: Bronckar, JP, Bulea, E. \& Bota, C. (éds). (éds). Le projet de Ferdinand de Saussure. Genève, Droz, 07-21.

Colombat, B., Fournier, J-M., Puech, C. (2010). Histoire des idées sur le langage et les langues. Paris: Klincksieck.

Constantin, E. (2005). Linguistique générale. Cours de m. le professeur F. de Saussure. Cahiers Ferdinand de Saussure, 58, 71-289.

Coseriu, E. (1980). Lições de linguística geral. Rio de Janeiro: Ao Livro Técnico.

Coseriu, E. (1982). Au délà du structuralisme. Actes, XVI Congrés Internacional de Lingüística Filologia Romàniques. Palma de Mallorca: Editorial Moll, 163-168.

Coseriu, E. (1982a). Mas alla del estructuralismo. San Juan: Faculdad de Filosofía, Humanidades y artes/Universidad Nacional de San Juan.

Coseriu, E. (1984). Fundamentos y tareas de la lingüística integral. Actas del Segundo Congreso Nacional de Lingüistica, 37-53.

Coseriu, E. (2007). Lingüistica del texto. Introducción a la hermenéutica del sentido (édition et annotation d'Oscar Loureda Lamas). Madrid: Arco/Libros.

Gambarara, D. (2005) Un texte original - présentation des textes de F. de Saussure. Cahiers Ferdinand de Saussure, 58, 29-42.

Hassler, G. (2015). La relation entre la philosophie du language et la sémantique chez Coseriu. In: Gérard, Christophe et Missire, Régis (eds). Eugenio Coseriu aujourd'hui - linguistique et philosophie du langage. Limoges: Lambert-Lucas, 21-33.

Jäger, L. (2013). La science du langage : les notes de l'orangerie et leur signification pour la théorie saussurienne du langage. Arena Romanistica, 12, 48-85.

Kabatek, J. (2003). Unidad del significado, designado y linguística integral. Odisea, n. 3, 87-99.

Kabatek, J. (2004). Eugenio Coseriu (1921-2002). Estudis Romànics. Vol. 26, 484-488.

Kabatek, J; Murguía, A. (1997). « Die Sachen sagen, wie sie sind... » Eugenio Coseriu im Gespräch. Tübingen: Gunter Narr.

Oancea, I.; Obrocea, N. (2013). Le centre d'études intégralistes de Cluj. Quelques repères. In: Bojoga, E.; Boc, O.; Vîlcu, D. C. (éditeurs). Coseriu: perspectives contemporaines. Tome 1. Cluj Napoca: Presa Universitara Clujeana. 
Pinheiro, C. L. (2016). Les recherches de Ferdinand de Saussure sur les légendes : en quel sen peuton parler d'etudes de textes ? In : Cruz, M. A. ; Piovezani, C. ; Testenoire, P-Y. Le discours et le texte : Saussure en héritage. L'Harmattan-Academia : Louvain-la-neuve, 181-194.

Robu, A. M. (2017). Interview with Prof. Dr. Johannes Kabatek. Anadiss - Revista de analiza discursului, JK hors serie, 139-149.

Saussure, F. (1916). Cours de linguistique générale. Paris : Payoy.

Saussure, F. (2002). Écrits de linguistique générale. Édition par Bouquet, S. et Engler, R. Paris: Gallimard.

Quijano, C. M. (2005). Sous le signe du doute - présentation des textes de E. Constantin. Cahiers Ferdinand de Saussure, 58, 34-67.

Testenoire, P-Y. (2016). Ce que les théories du discours doivent à Saussure : note sur la note « sur le discours ». In : Cruz, M. A. ; Piovezani, C. ; Testenoire, P-Y. Le discours et le texte : Saussure en héritage. L'Harmattan-Academia : Louvain-la-neuve, 109-131. 